\title{
The Classification of Finite Simple Groups: A Progress Report
}

Ronald Solomon

Communicated by Alexander Diaz-Lopez and Harriet Pollatsek

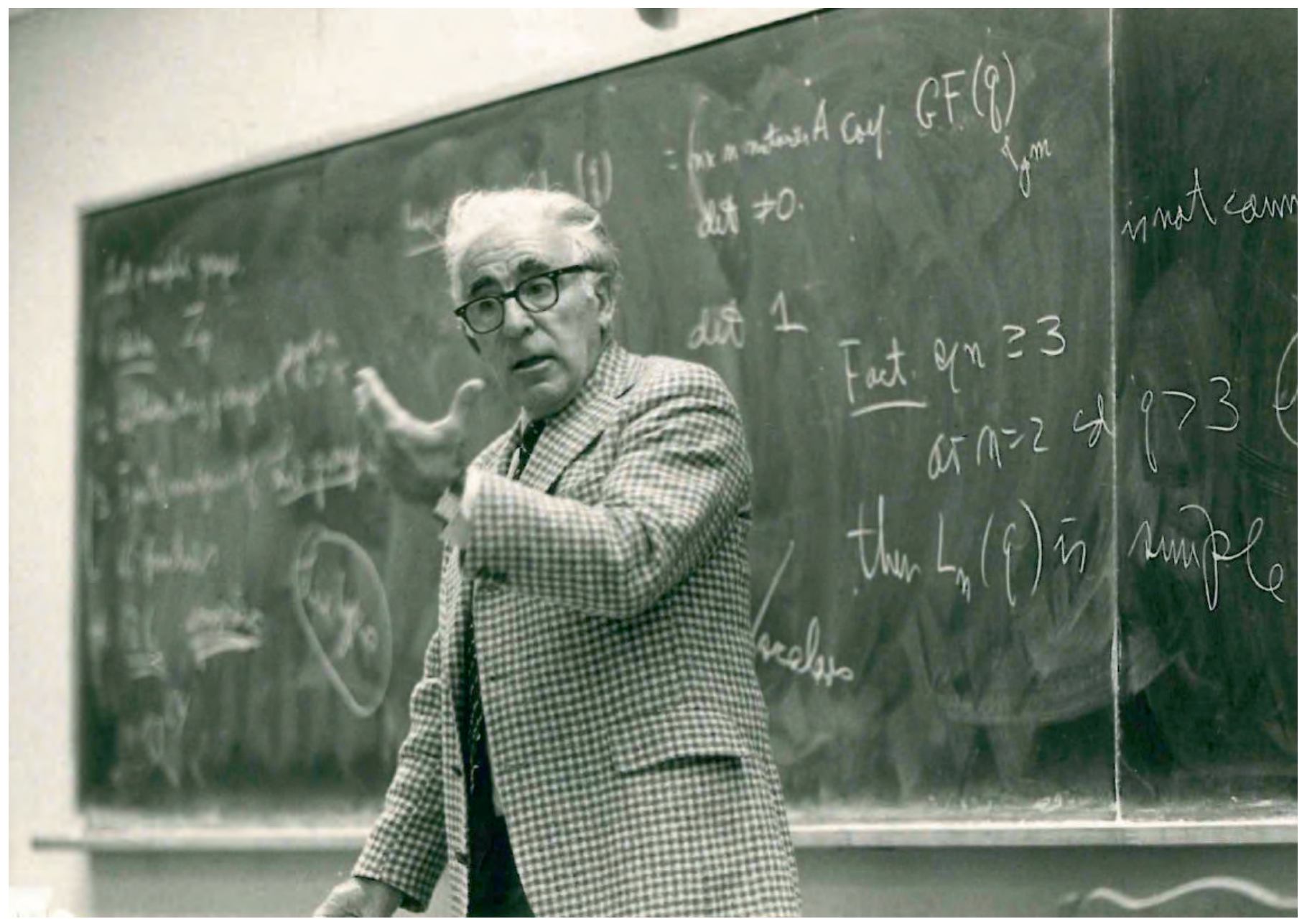

Danny Gorenstein dubbed the classification of finite simple groups "The Thirty Years' War."

Ronald Solomon is professor emeritus at the Ohio State University.

His email address is solomon@math. ohio-state. edu.

For permission to reprint this article, please contact:

reprint-permission@ams.org.

DOI: http://dx.doi.org/10.1090/noti1689 


\section{History of the Project}

In 1981 the monumental project to classify all of the finite simple groups appeared to be nearing its conclusion. Danny Gorenstein had dubbed the project the "Thirty Years' War," dating its inception from an address by Richard Brauer at the International Congress of Mathematicians in 1954. He and Richard Lyons agreed that it would be desirable to write a series of volumes that would contain the complete proof of this Classification Theorem, modulo a short and clearly specified list of background results. As the existing proof was scattered over hundreds of journal articles, some of which cited other articles that were never published, there was a consensus that this was indeed a worthwhile project, and the American Mathematical Society (AMS) agreed to publish this series of volumes. In the spring of 1982, Danny and Richard recruited me to be a partner in this project. Richard Foote and Gernot Stroth were also recruited at an early stage to contribute specific portions of this work.

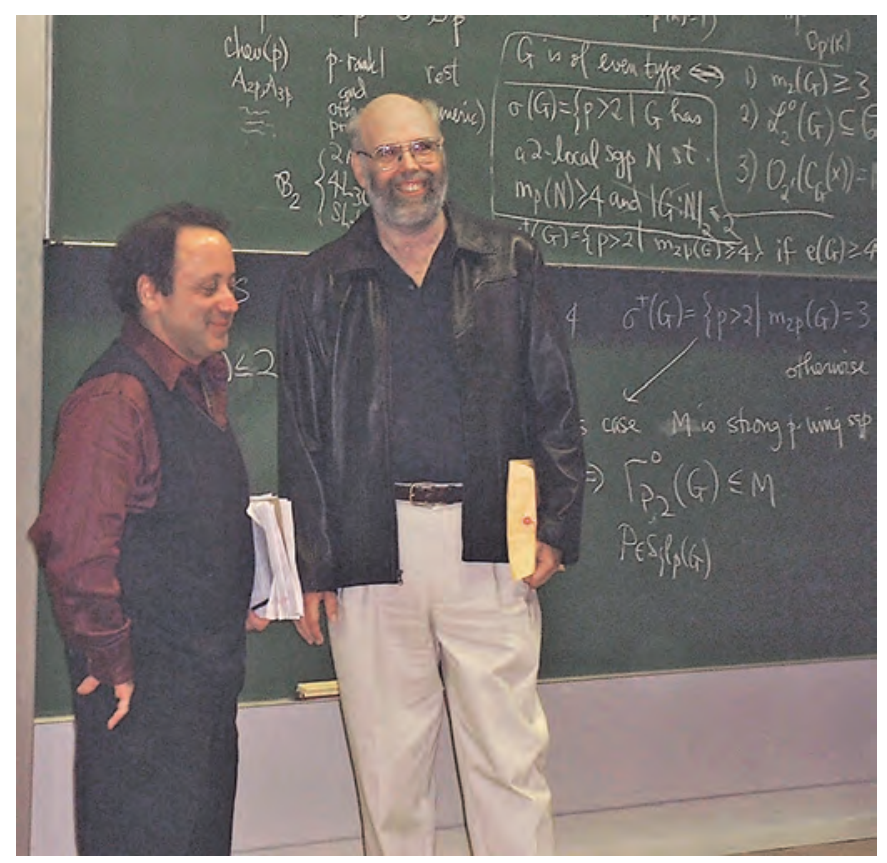

Richard Lyons (pictured here with the author) and Gorenstein agreed that it would be desirable to write a series of volumes [GLS] that would contain the complete proof of this Classification Theorem.

Considerable progress was made during the first decade of the project, and then, tragically, Danny Gorenstein died in August 1992. Nevertheless, the first six volumes of our series were published by the AMS during the decade 1994-2005. Then a hiatus ensued. I am happy to report that Volume 7 has just been published by the AMS, and Volume 8 is near completion and promised to the AMS by August 2018. The completion of Volume 8 will be a significant mathematical milestone in our work. It seems a good time to provide this progress report on the GLS project. Moreover, although the strategy outlined in Volume 1 [GLS] remains substantially unchanged, there is one significant change worthy of note. (A detailed overview of the original Classification Project may be found in [ALSS].)

We anticipate that there will be twelve volumes in the complete series [GLS], which we hope to complete by 2023. I will report on the current state of our project in this article.

\section{Introduction to Classification}

A common theme in mathematics is to study a particular mathematical structure and attempt to classify all instances of it, e.g., regular polyhedra, distance transitive graphs, and 3-manifolds, among others. Classifying infinite groups is hopeless, but already in the 1890s, inspired by the work of Killing and Cartan on finite-dimensional semisimple complex Lie algebras, some mathematicians began to contemplate the classification of all finite groups.

Can we classify all finite groups? Since we can combine any two finite groups, for instance via direct product, to create a new finite group, it is natural to begin by considering groups that are "building blocks" for all other finite groups. Similar to the factorization of integers into prime numbers, one can "break down" finite groups into smaller pieces called simple groups, which cannot be decomposed further. A group $G$ is simple if it has no nontrivial proper normal subgroup; i.e., its only normal subgroups are the trivial group and $G$ itself. The Jordan-Hölder Theorem then tells us that for any finite group $G$, there is an ordered sequence of subgroups, $1=H_{1} \triangleleft H_{2} \triangleleft \cdots \triangleleft H_{n}=G$ called a composition series, such that each $H_{i}$ is a maximal proper normal subgroup of $H_{i+1}$ and all $H_{i+1} / H_{i}$, called composition factors, are simple. Moreover, any two composition series of a group $G$ are equivalent in the sense that they have the same number of subgroups and the same composition factors, up to permutation and isomorphism.

Nonetheless, the problem of determining all ways to reassemble a set of composition factors into a finite group is daunting, perhaps infeasible. Although it is very easy to prove that the only abelian simple groups are $\mathbb{Z} / p \mathbb{Z}$, where $p$ is a prime number, the problem of determining all finite $p$-groups, i.e., groups $G$ all of whose composition factors are isomorphic to $\mathbb{Z} / p \mathbb{Z}$, is of frightening complexity. For example, there are billions of groups of size $2^{10}$, and many of them are essentially indistinguishable.

Fortunately, in most applications of finite group theory, where the group arises as a group of permutations or symmetries or linear operators on some other structure, the problem can be reduced easily to the case where the group action is "primitive" in some sense. Using the classification of finite simple groups, Bob Guralnick, the latest Cole Prize recipient (see Notices, April 2018, p. 461), has demonstrated the efficacy of this strategy in a wide variety of contexts.

For the remainder of this article, I will discuss the classification of the finite simple groups. In contrast to the abelian case, the classification of nonabelian finite simple groups is quite complex and requires a fullscale classification strategy. The two smallest nonabelian 
simple groups are $A_{5}$, the alternating group on five symbols, with 60 elements, and PSL $(2,7)$, a member of one of the families of groups of Lie type (see the next section), with 168 elements. In addition to the simple groups belonging to infinite families, there are also twenty-six so-called sporadic groups, five discovered by Mathieu in the nineteenth century, and the rest discovered between 1965 and 1975. The sporadic groups range in size from 7920 (the smallest Mathieu group) to approximately $8 \times 10^{53}$ (the aptly named Monster).

In the next section, we discuss our classification strategy to prove the following theorem.

Theorem. Every finite simple group is isomorphic to one of the following:

(1) a cyclic group of prime order,

(2) an alternating group of degree at least 5 ,

(3) a simple group of Lie type, or

(4) one of the 26 "sporadic" simple groups.

\section{The Classification Strategy}

For about fifty years, the Classification Strategy has been schematically represented as a box subdivided into four smaller boxes:

\begin{tabular}{|l|l|}
\hline small odd & small even \\
\hline large odd & large even \\
\hline
\end{tabular}

Most of the finite simple groups are groups of Lie type defined over some finite field. If you are not familiar with Lie groups, you may consider the example of $\operatorname{PSL}(n, F)$, which is the quotient of the group $\operatorname{SL}(n, F)$ of $n \times n$ matrices of determinant 1 with entries from the finite field $F$ by the normal subgroup of scalar matrices of determinant 1 . In this context, odd and even refer to the parity of $|F|$, while small and large are measured by the size of $n$. When $n<3$, the groups are definitely small, though $\operatorname{PSp}(4, F)$ is also small. If you know Tits's description of groups of Lie type as BN pairs, then it is more accurate to say that groups of BN rank 1 or 2 are small, while most groups of BN rank at least 3 are large.

Of course, there are also simple alternating groups and sporadic groups which must be fitted into this scheme. Much more serious is the fact that we must provide definitions for terms like "a group $G$ of small odd type" that do not presuppose that $G$ is a group of Lie type or indeed has any known property other than simplicity. However, if $p$ is a prime divisor of $|G|$, then Sylow guarantees the existence of many $p$-subgroups of $G$, i.e., subgroups of order $p^{m}$ for some $m \geq 1$. We call the centralizers and normalizers of such subgroups $p$-local subgroups of $G$. In his 1954 address, Richard Brauer made the case for attempting to characterize simple groups via their 2-local structures. A crucial validation for this strategy was provided in 1963 when Walter Feit and John G. Thompson [FT] published their proof that all nonabelian finite simple groups have even order.

Now, if $G=G(F)$ is a group of Lie type defined over a field $\mathrm{F}$ of even order, then the 2-local subgroups of $G$ are contained in parabolic subgroups of $G$, and by a theorem of Borel and Tits they inherit significant structural properties from these parabolics. On the other hand, this is far from true in groups of Lie type when $F$ has odd order, with a small number of interesting exceptions. This gave rise to the initial definitions of "even" and "odd": characteristic 2-type and "non"-characteristic 2-type. (We shall instead call the latter "odd type.")

Definition. A group $H$ is of 2-parabolic type if $H$ contains a normal 2-subgroup $Q$ such that the centralizer $C_{H}(Q)$ is just $Z(Q)$. (This definition is nonstandard. It is used here for expository purposes.)

Definition. A group $G$ is of characteristic 2-type if every 2-local subgroup of $G$ is of 2-parabolic type.

A weakness of using this definition to define the line between even and odd is that, in the study of groups of odd type, it forces the consideration of all groups $G$ having a 2-local, no matter how small, which is not of 2-parabolic type. A better definition is

Definition. A group $G$ is of even characteristic (or parabolic characteristic 2 ) if every 2 -local subgroup $H$ of odd index in $G$ is of 2-parabolic type.

In the GLS Project, we use another term, "even type," which is convenient for our approach but technically complicated to define. Recently, Magaard and Stroth have classified all groups that are of even type but not of even characteristic. The list is rather short: $A_{12}, \Omega_{7}(3)$, $\Omega_{8}^{-}(3)$, and the sporadic simple groups $J_{1}, \mathrm{Co}_{3}$, and $\mathrm{Fi}_{23}$. I shall speak henceforth of the odd/even dichotomy as the dichotomy between groups of even type and groups of odd (i.e., not even) type.

The small/large dichotomy was first formulated for groups of even type by John G. Thompson in his work on $N$-groups, i.e., nonsolvable finite groups all of whose local subgroups are solvable. He defined the parameter $e(G)$ to be the maximum rank of an abelian subgroup of odd order contained in some 2-local subgroup of $G$. A finite simple group $G$ is of small even type if $e(G) \leq 2$, i.e., if no 2-local subgroup $H$ of $G$ contains a subgroup isomorphic to $C_{p} \times C_{p} \times C_{p}$ for any odd prime $p$. Such groups also came to be known as quasithin groups, and the classification of quasithin simple groups of even characteristic by Aschbacher and Smith [AS] was the culminating accomplishment of the original Classification Project. Notice that if $G$ is a Chevalley group defined over a field of even order $q>2$, then $e(G)$ typically measures the rank of a split torus of $G$ and hence the BN-rank of $G$.

The small/large dichotomy for groups of odd type was originally formulated in terms of the 2-rank (or normal 2-rank or sectional 2-rank) of $G$. In the [GLS] volumes, the study of groups $G$ of odd type focuses on the isomorphism types of components of involution centralizers, where an involution is an element of order 2 in $G$. The following definitions are of crucial importance.

\section{Definitions.}

1. A finite group $L$ is quasisimple if $L / Z(L)$ is a nonabelian simple group and $L=[L, L]$. 


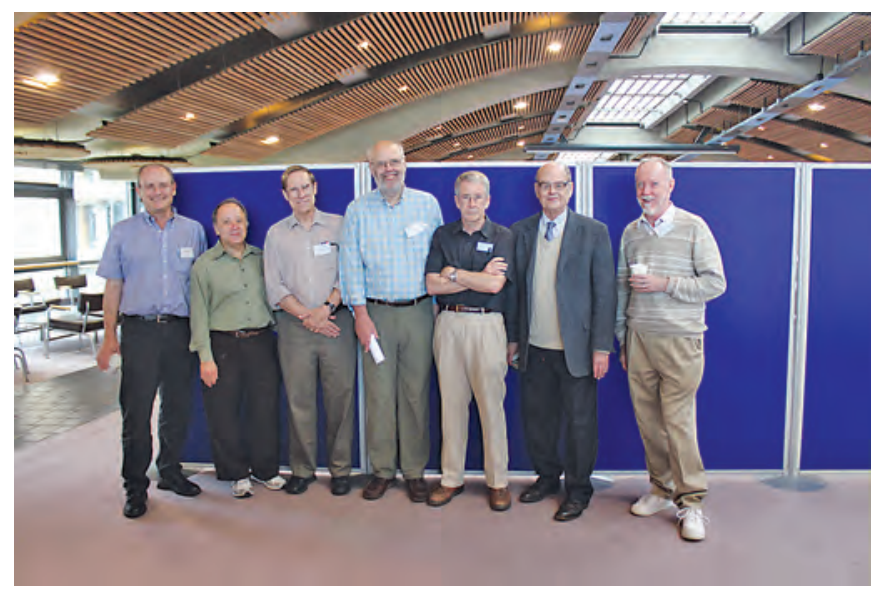

Some of those who have worked on the Classification Project (I to r: David Goldschmidt, Ron Solomon, Stephen D. Smith, Richard Lyons, Michael Aschbacher, John G. Thompson, and Richard Foote), who gathered at Cambridge University in 2013 in celebration of Thompson's eightieth birthday.

2. A subgroup $L$ of a group $H$ is a component of $H$ if $L$ is a quasisimple subnormal subgroup of $H$. (Subnormality is the transitive extension of the normality relation.)

If, for example, $G=\mathrm{GL}(V)$, where $V$ is an $n$-dimensional vector space over a finite field $F$ of odd order, and if $t$ is an involution in $G$, then the components of $C_{G}(t)$ typically are $\operatorname{SL}\left(V_{-}(t)\right)$ and $\operatorname{SL}\left(V_{+}(t)\right)$, where $V_{-}(t)$ and $V_{+}(t)$ are the -1 and +1 eigenspaces for $t$ on $V$. Thus the components of $C_{\mathrm{GL}(V)}(t)$ are $\left[C_{+}, C_{+}\right] \cong \mathrm{SL}\left(V_{+}\right)$and $\left[C_{-}, C_{-}\right] \cong \operatorname{SL}\left(V_{-}\right)$. See Figure 1. (Computations are similar in $\operatorname{SL}(V)$ and $\operatorname{PSL}(V)$, and the components are isomorphic to those in $\mathrm{GL}(V)$.)

$$
\begin{gathered}
t=\left(\begin{array}{cc}
I & O \\
O & -I
\end{array}\right) \\
C_{\mathrm{GL}(V)}(t)=\left\{\left(\begin{array}{cc}
A & O \\
O & B
\end{array}\right): A \in \mathrm{GL}\left(V_{+}\right), B \in \mathrm{GL}\left(V_{-}\right)\right\} \\
=C_{+} \times C_{-} \cong \operatorname{GL}\left(V_{+}\right) \times \operatorname{GL}\left(V_{-}\right) .
\end{gathered}
$$

Figure 1. The components of $C_{\mathrm{GL}(V)}(t)$ are $\left[C_{+}, C_{+}\right] \cong \operatorname{SL}\left(V_{+}\right)$and $\left[C_{-}, C_{-}\right] \cong \operatorname{SL}\left(V_{-}\right)$.

Roughly speaking, groups of small odd type are defined to be simple groups $G$ such that the only components occurring in any involution centralizer are isomorphic to $\operatorname{SL}(2, F)$ or $\operatorname{PSL}(2, F)$ for some field $F$ of odd order. The classification of finite simple groups of small odd type appears in Volume 6 [GLS]. Since the [GLS] Project will quote [AS] for the classification of quasithin groups of even type, 2004 marked the publication of the volumes treating "small" groups of both odd and even type.

It should be noted here that in the outline of the [GLS] series in Volume 1, a slightly broader definition of small even type was formulated. Namely, it was stipulated that $G$ was of small even type if $G$ was of even type with $e(G) \leq 3$ and no 2-local subgroup $H$ of $G$ contained a subgroup isomorphic to $C_{p} \times C_{p} \times C_{p}$, unless $p=3$. It was anticipated that the Quasithin Problem would be enlarged to treat this wider problem. Given the monumental task accomplished by Aschbacher and Smith, it is quite understandable that they limited themselves to the original Quasithin Problem (though extended to groups of even type). It should also be noted that both Aschbacher-Smith [AS] and [GLS] treat only $\mathcal{K}$-proper simple groups, i.e., simple groups all of whose proper simple sections are known simple groups. This certainly suffices for an inductive proof of the Classification Theorem.

In the philosophy of the GLS Project, large simple groups of both even and odd type are to be treated as groups of component type, i.e., groups $G$ that, for a suitable prime $p$, have $p$-rank at least 3 and have elements of order $p$ whose centralizers have components of suitable type. Notice that if $G$ is a simple group of Lie type defined over a field $F$ of characteristic $r$, then centralizers of elements of order $r$ have no components, while elements of prime order $p$ different from $r$ often do. For example, if $G=\mathrm{GL}(6, F),|F|=2^{2 m}$, and $x$ is an element of $G$ of order 3 with three 2-dimensional eigenspaces, with eigenvalues $1, \omega$, and $\omega^{2}$, then $C_{\mathrm{GL}(6, F)}(x)$ has three components, each isomorphic to $\operatorname{SL}(2, F)$ as in Figure 2. Again, the calculations are similar in $\operatorname{SL}(6, F)$ and in $\operatorname{PSL}(6, F)$.

$$
x=\left(\begin{array}{ccc}
I & O & O \\
O & \omega I & O \\
O & O & \omega^{2} I
\end{array}\right)
$$

and

$$
\begin{aligned}
C_{\mathrm{GL}(6, F)}(x) & =\left\{\left(\begin{array}{lll}
A & O & O \\
O & B & O \\
O & O & C
\end{array}\right): A, B, C \in \mathrm{GL}(2, F)\right\} \\
& \cong \mathrm{GL}(2, F) \times \mathrm{GL}(2, F) \times \mathrm{GL}(2, F) .
\end{aligned}
$$

Figure 2. The centralizer $C_{\mathrm{GL}(6, F)}(x)$ of an order-3 element $x$ has three components, each isomorphic to $\operatorname{SL}(2, F)$. Here $|F|=2^{2 m}$.

In general, in groups $G$ of characteristic $r$, centralizers of elements of order $p$ will typically have components that themselves are quasisimple groups of Lie type of characteristic $r$, indeed often smaller dimensional versions of $G$ itself, as in the example in Figure 2. Thus, the principal criterion for "components of suitable type" is that they not be groups of Lie type of characteristic $p$. In this case, we shall call $p$ a semisimple prime for $G$.

More accurately, we choose $p=2$ if some involution centralizer $C_{G}(t)$ has a component which is not a group of Lie type in characteristic 2 . We also exclude most sporadic components and, for the sake of "largeness," $\mathrm{SL}_{2}(q)$ and $\mathrm{L}_{2}(q)$. If we cannot choose $p=2$, then we choose an odd prime $p$ such that $G$ has $p$-rank at least 3 and some $p$-element centralizer $C_{G}(x)$ has a component which has a noncyclic Sylow $p$-subgroup and is not a group of Lie type in characteristic $p$. (For $p=3$, we also exclude several sporadic components.) 
I have swept under the carpet a serious problem concerning " $p$-signalizers," and I shall continue to do so. But I will say that I should be saying " $p$-components" and not "components" in the previous paragraph, and the proof that we may reduce the problem from $p$-components to components when either $p=2$ or $p$ is odd and $G$ has $p$-rank at least 4 is one of the principal results of Volume 5 [GLS]. For this reason, we use the term generic even type to refer to the subset of groups of large even type which have $p$-rank at least 4 for a suitable odd prime $p$. The signalizer problem for groups of characteristic 2-type and odd $p$-rank 3 was handled by Aschbacher in a pair of papers, which still need to be generalized to the case of groups of even type.

Building on the work in Volume 5 [GLS], Volumes 7 and 8 will complete the proof of the following two theorems.

Theorem 0 . Let $G$ be a finite $\mathcal{K}$-proper simple group of odd type. Then either $G$ is an alternating group of degree $n \geq 5$ (but not 8 or 12) or $G$ is a group of Lie type defined over a finite field of odd order or $G$ is one of the following sporadic simple groups: $M_{11}, M_{12}, J_{1}, M c, O^{\prime} N$, or Ly.

Theorem GE. Let $G$ be a finite $\mathcal{K}$-proper simple group of generic even type. Then either $G$ is a group of Lie type defined over a finite field of even order or $G$ has a proper $p$-uniqueness subgroup for some odd prime $p$ or $G$ has a " $p$-thin configuration" for some odd prime $p$.

Volume 7 [GLS] has just been published by the AMS. It almost completes the identification of the alternating groups of degree $n \geq 13$ and the reduction of the Generic Case to the case where a suitable $p$-element centralizer has a component of Lie type in characteristic $r$, with either

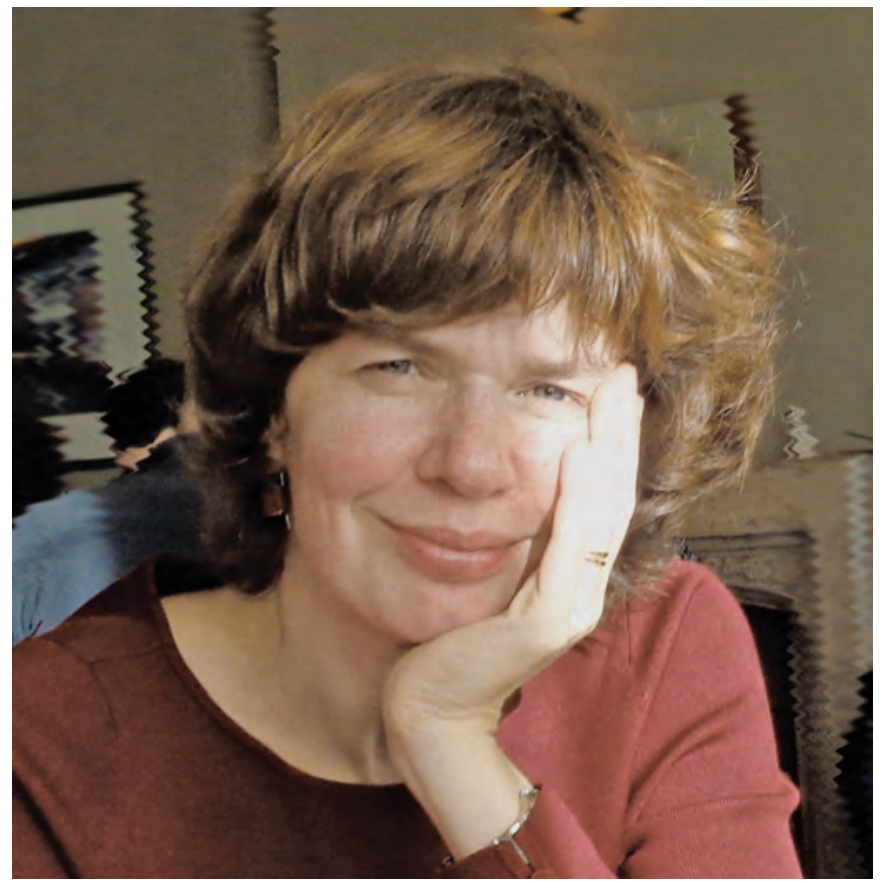

Inna Capdeboscq, coauthor of Volume 9 [GLS].

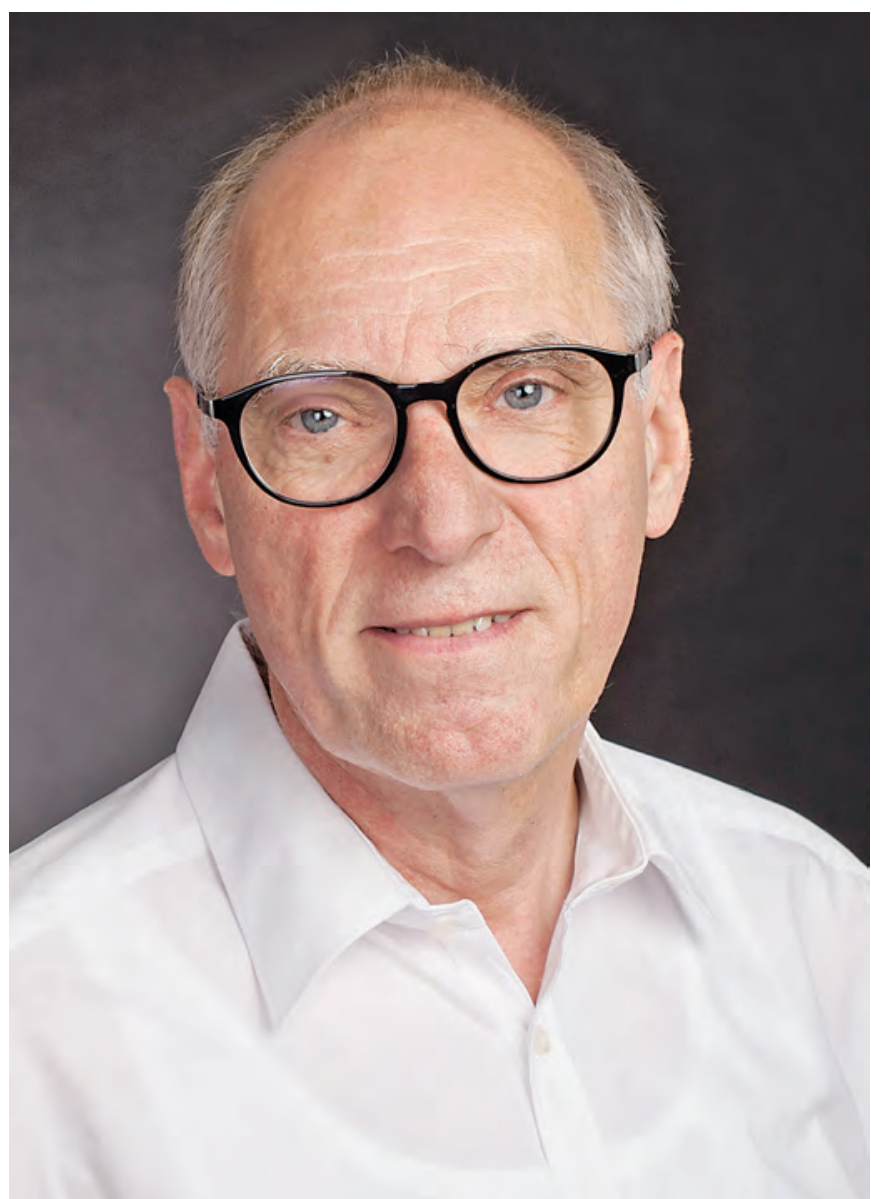

Gernot Stroth's 600-page treatment of groups with a strongly $p$-embedded subgroup will probably appear as Volume 11 [GLS].

$p=2$ or $r=2$. Volume 8 will complete the identification of the alternating groups and the generic groups of Lie type. It is near completion, and we anticipate submission to the AMS for publication by August 2018.

An important project stimulated by our work was the study of a class of amalgams of finite groups, known as Curtis-Tits-Phan amalgams, by a team of mathematicians including C. Bennett, R. Blok, R. Gramlich, C. Hoffman, M. Horn, B. Muhlherr, W. Nickel, and S. Shpectorov. Their results are added to our list of Background Results and used in our identification of many of the finite simple groups of Lie type. An extensive overview of this project, with a fuller list of authors and papers, may be found in Gramlich [Gr]. A very recent paper of Blok, Hoffman, and Shpectorov is also crucial to our work.

What will remain to be done after Volume 8 [GLS]? The remaining work falls primarily into three cases:

(1) the Bicharacteristic Case,

(2) the $p$-Uniqueness Case, and

(3) the $e(G)=3$ Case.

The Bicharacteristic Case refers to the case when $G$ is of even type with $e(G) \geq 4$ but there is no semisimple prime 
p. Thus, $G$ seems to have two different characteristics: 2 and $p$. Using an argument of Klinger and Mason, it is easy to see that $p=3$. This is a phenomenon that occurs in many sporadic simple groups as well as a few groups defined over fields of order 2 or 3 . Considerable work has been done on this problem, originally by Gorenstein and Lyons, and more recently by Inna Capdeboscq, Lyons, and me. We anticipate that this will be the principal content of Volume 9 [GLS], coauthored with Capdeboscq. This volume will also dispose of the dangling $p$-Thin case from Theorem GE.

The $p$-Uniqueness Case arises when the proof of Theorem GE leads to the construction of a proper subgroup $M$ of $G$ containing all (or almost all) of the $p$-local overgroups of a fixed Sylow $p$-subgroup of $G$. When $p=2$, this problem was handled elegantly by Helmut Bender and Aschbacher, and their work is contained in Volume 4 [GLS]. (Similarly, the 2-Thin Problem was handled in Volume 6, which explains why the statement of Theorem $\mathrm{O}$ is so much cleaner than the statement of Theorem GE.) When $p$ is odd, there is a major 600-page manuscript by Gernot Stroth treating groups with a strongly $p$-embedded subgroup, which will appear in the [GLS] series, probably in Volume 11. There are also substantial drafts by Richard Foote, Gorenstein, and Lyons for a companion volume (Volume 10?), which together with Stroth's volume will complete the $p$-Uniqueness Case. In particular, Volumes 9, 10, and 11 will strengthen Theorem GE to

Theorem GE+. Let $G$ be a finite $\mathcal{K}$-proper simple group of generic even type. Then $G$ is a group of Lie type defined over a finite field of even order.

There remains the gap between generic even type and large even type, i.e., the $e(G)=3$ Case. If $G$ is a $\mathcal{K}$-proper simple group of characteristic 2-type with $e(G)=3$, then $G$ was classified in a pair of papers by Michael Aschbacher. The first paper treats the case when a 2-local $H$ contains an abelian $p$-subgroup $A$ of rank 3 for some $p>3$. Aschbacher's method in this case should extend fairly easily to our case, i.e., the case when $G$ is of even type. In the second paper, 3 is the only choice for $p$. This is the case that was redefined as a subproblem of the Quasithin Problem in Volume 1 [GLS]. It has been given serious attention by several of our colleagues, notably Capdeboscq and Chris Parker, who have a manuscript overcoming one of the difficulties of extending Aschbacher's work to the even type situation. In this context, it might be very helpful to have a proof of the following result:

Statement. Let $G$ be a finite group with an abelian Sylow $p$-subgroup $P$. Suppose that $P$ contains a strongly closed elementary abelian subgroup $B$. Then $G$ contains a normal subgroup $N$ such that $B=\left\langle x \in P \cap N: x^{p}=1\right\rangle$.

Using the Classification Theorem, this result is a special case of a theorem of Flores and Foote. However, we are not permitted to quote the Classification Theorem. For the purposes of our problem, it would suffice to assume that $p=3$ and $P$ has 3-rank 3 .
It would be wonderful to complete our series by 2023 , the sixtieth anniversary of the publication of the Odd Order Theorem. Given the state of Volumes 8, 9, 10, and 11 , the achievement of this goal depends most heavily on the completion of the $e(G)=3$ problem. It is a worthy goal.

\section{Image Credits}

Photo of Danny Gorenstein and group photo of Classification Project participants courtesy of Richard Foote.

Photo of Richard Lyons and Danny Gorenstein by Richard Foote. Figures 1 and 2 by author.

Photo of Inna Capdeboscq by Yves Capdeboscq.

Photo of Gernot Stroth courtesy of Gernot Stroth.

Author photo by Rose Solomon.

\section{References}

[ALSS] M. Aschbacher, R. Lyons, S. D. SMith, and R. SOlOmon, The Classification of Finite Simple Groups, Mathematical Surveys and Monographs Vol. 172, Amer. Math. Soc., 2011. MR2778190

[AS] M. ASCHBACHER and S. D. SMITH, The Classification of Quasithin Groups I, II, Mathematical Surveys and Monographs 111, 112, Amer. Math. Soc., 2004. MR2097623, MR2097624

[FT] W. FeIT and J. G. Thompson, Solvability of groups of odd order, Pacific J. Math. 13 (1963), 775-1029. MR0166261

[GLS] D. GORENSTEIN, R. LyONS, and R. SOlOMON, The Classification of the Finite Simple Groups, Mathematical Surveys and Monographs 40, 1-7, Amer. Math. Soc., 1994, 1996, 1998, 1999, 2002, 2005, 2018. MR1303592, MR1358135, MR1490581, MR1675976, MR1923000, MR2104668

[Gr] R. GRAmLICH, Developments in finite Phan theory, Innov. Incidence Geom. 9 (2009), 123-175. MR2658896

\section{ABOUT THE AUTHOR}

Ronald Solomon first learned to love words from his mother; to love math from his geometry teacher, Blossom Backal; and to love classical music from Mozart's Great G minor Symphony. He is the proud husband of Rose, father of Ari and Michael, and grandfather of Sofia. 